\title{
The willingness of general practitioners to be involved in the follow-up of adult survivors of childhood cancer
}

\author{
Ria Blaauwbroek • Nynke Zwart • \\ Martijn Bouma • Betty Meyboom-de Jong • \\ Willem A. Kamps • Aleida Postma
}

Received: 18 July 2007 / Accepted: 24 August 2007 / Published online: 27 September 2007

(C) Springer Science + Business Media, LLC 2007

\begin{abstract}
Background Long-term follow-up of childhood cancer survivors is mainly organised by paediatric oncologists and until now general practitioners (GPs) are rarely involved. To ensure appropriate follow-up for all survivors into adulthood, a combined effort of paediatric oncologists and general practitioners might be the solution. We investigated the willingness of GPs, who had followed a postgraduate course on late effects of cancer treatment, to participate in a shared care model for follow-up of adult childhood cancer survivors as well as what their requirements would be in case of participation.

Methods From the Northern Netherlands, 358 GPs participated in a postgraduate course on late effects in paediatric cancer survivors. After the course, they were asked to complete a 10-item questionnaire on motivation to participate in the regular follow-up of adult childhood cancer survivors as well as their conditions to participate.

Results The response rate was $65 \%$. Of the responders, $97 \%$ were willing to participate in a shared care model for follow-up and $64 \%$ felt that it was their responsibility to be in charge of childhood cancer survivors. The main require-
\end{abstract}

\footnotetext{
R. Blaauwbroek $(\bowtie) \cdot$ N. Zwart $\cdot$ M. Bouma •

W. A. Kamps • A. Postma

Department of Paediatrics, Division of Paediatric Oncology, University Medical Centre Groningen, University of Groningen, Hanzeplein 1, P.O. Box 30 001, 9700 RB Groningen,

The Netherlands

e-mail: r.blaauwbroek@bkk.umcg.nl

\section{B. Meyboom-de Jong}

Department of General Practice,

University Medical Centre Groningen, University of Groningen, Antonius Deusinglaan 1,

9713 AV Groningen, The Netherlands
}

ments for participation were the availability of guidelines (64\%), sufficient information about the patient's medical history (37\%), and short communication lines (45\%). The main barriers to participate were workload (16\%), lack of knowledge (15\%), and lack of communication (13\%).

Conclusion A significant number of GPs are ready to participate in the long-term follow-up of adult childhood cancer survivors if adequate guidelines and medical information is provided and communication lines are clear.

Keywords Childhood cancer survivors ·

Long-term follow-up · General practitioners

\section{Introduction}

One of the growing challenges in medicine is providing appropriate health care for survivors of childhood and adolescent cancer. Childhood cancer is rare but major advances in paediatric cancer therapy have led to long-term survival of approximately $75 \%$ of children treated [1]. This success story has been tempered by the knowledge that cure has often come at a price, which may not be manifest until many years after therapy. Late or long-term effects are defined as persistent and adverse changes due to the disease and/or its treatment. Because childhood is a time of rapid physical growth and emotional development, the consequences of therapy can be far different from the results of similarly treated adults. It is estimated that physical and/or psychosocial complications may develop in as many as two-thirds of these young adults [2].

Late effects vary in severity dependent on the specific type of cancer, treatment received, and the age of the child during therapy. 
Because the population of adult survivors of childhood cancer is still relatively young, with only a small portion over the age of 40 , there are only few data yet to answer the question if survivors of childhood cancer are at risk for prevalent diseases of middle to later life. In an earlier study we showed that the prevalence and the severity of late effects increased with time since diagnosis [3]. As time since diagnosis extends, medical problems associated with aging may exhibit an earlier onset or more accelerated course following certain cancer therapies. Oeffinger et al. [4] described that cancer survivors, diagnosed with cancer between 1970 and 1986, were more vulnerable to diseases that are associated with aging, like second cancers, heart conditions, kidney disease, musculoskeletal problems, osteoporosis, and sterility compared with their siblings.

Only through long-term follow-up of adult survivors the impact of these types of iatrogenic late effects on the aging process will become evident, and thus we will be able to rationally determine long-term risk-benefit ratios. A systematic plan for lifelong screening, surveillance, and prevention that incorporates risks based on the previous cancer, cancer therapy, genetic predispositions, lifestyle behaviours, and co-morbid health conditions should be developed for all survivors.

The improvements in outcome have not been accompanied by equal progress in the manner in which care is provided to (young) adults with these conditions. It has been reported that less than $20 \%$ of adult survivors of childhood cancer are followed at a cancer centre or by an oncologist. As the number of survivors of childhood cancer is expected to increase further, there is some urgency in determining where long-term follow-up should take place.

It will be difficult for the usual paediatric oncology clinical services to accommodate the demands of the everenlarging population of survivors. Moreover, adult survivors do not fit in paediatric clinics, thus transition of care from the paediatric to the adult health care setting is necessary for most childhood cancer survivors. By integrating general practitioners in the paediatric programs, a seamless transition from a predominantly paediatric- to a predominantly adult-oriented follow-up can be guaranteed.

Currently a typical general practitioner's practice would include about two to three adult survivors, but as the number of childhood cancer survivors increases, general practitioners will encounter childhood cancer survivors in their practices more often, up to eight to nine in 2010 [5]. These survivors are a heterogeneous population with a variety of different cancers diagnosed at different age periods or different treatment protocols and recommendations for screening are continuously evolving. Thus it is understandable that general practitioners are not particularly aware of the risks of this population. Although there is considerable information available about long-term effects, most has been published in the paediatric or oncology literature. It is important to establish systems by which general practitioners become knowledgeable about the late sequels of cancer treatment and its consequences on adult health. Information materials need to reach primary care providers before their first interaction with a patient who is a childhood cancer survivor. The aim of this study was to investigate the views and needs of general practitioners on participating in a shared care program for the follow-up of adult survivors of childhood cancer.

\section{Methods}

Bi-annual refresher courses have been organised for GPs in the Northern provinces of The Netherlands for more than 30 years. These 5 days courses are repeated for 3 or 4 weeks in succession; each course is attended by about 50 GPs. The program for these courses is defined by a committee of GPs. The courses invite lecturers who are experts in the selected subjects. These courses have established a good reputation and are attended by about two-third of all GPs in these provinces. One week prior to the onset of the course the attending GPs receive a syllabus on the specific topics. In 2005 and in 2006 a paediatric oncologist and a GP working at the Long-term Follow-up clinic (LFTU) of the UMC Groningen were invited to lecture on adverse late effects in childhood cancer survivors.

Late effects of treatment after childhood cancer were explained using case histories of childhood cancer survivors (Table 1). Shared care of paediatric oncologists and patient's own GPs was discussed as a possible model for the long-term follow-up of childhood cancer survivors. Low frequent LFTU clinic are combined with regular follow-up in the GPs practice. The screening in the GPs practice will take place after the GP had been extensively informed about the previous history and health risks by the LFTU clinic. GPs were requested to evaluate the lecture using a 1-5 scale [(bad)-(excellent)]. At the end of the course all 358 GPs received an envelop containing a 10item questionnaire (Table 2) and were asked to return it by mail within 2 weeks to assess their opinion about shared care as a future plan for the follow-up of adult childhood cancer survivors. They were asked what their main requirements and barriers were. Also questions were asked about compensation, electronical forms and the role of a practice assistant in the screening process. These answers were plotted on a seven-point scale (most important- not important at all). The answers on the open questions 'If you were asked to participate in a shared-care program for the follow-up of adult childhood cancer survivors what would your requirements be?' and 'If you were asked to 
Table 1 Content of lecture on late effects in childhood cancer survivors

Content

Introduction

Case A: A 31-year-old man treated for Hodgkin Disease at age 12 with chest pain

Case B: A pregnant woman treated for Ewing Sarcoma at age 2 with i.a. anthracyclines, who desires to deliver at home

Case C: A 35-year-old woman treated for childhood cancer with irregular menstrual cycle

Case D: A 27-year-old man treated for ALL at age 7 with cranial radiation and now having epileptic insults

Case D: Same case as above 2 years later presenting with fatigue and metabolic syndrome

The need for long-term follow-up and the role of the GP
Most common adverse late effects after chemotherapy treatment in childhood cancer survivors

Most common adverse late effects after radiation therapy in childhood cancer survivors

Coronary artery disease after mediastinal radiation

Cardiotoxicity after treatment with anthracyclines

Immature ovarian failure and premature menopause

Risk of second malignancies

Growth hormone deficiency and risk for metabolic syndrome

Low frequent controls in the LFTU clinic, combined with regular follow-up in the GPs practice as a new model for long-term follow-up participate in a shared-care program for the follow-up of adult childhood cancer survivors what would your reservations be?' were categorized in seven respectively six different categories (Figs. 1 and 2).

Data were analyzed using SPSS 14 which generated descriptive statistics.

\section{Results}

In 2005 and 2006, 358 GPs out of a total of 507 (71\%) from two northern provinces in the Netherlands attended the refresher course. The response rate was $65 \%$ (233 out of 358 questionnaires; Table 2). The overall lecture was evaluated as an mean 4.51(SD 0.54) for the content and an mean 4.23 (SD 0.56) out of 5 for the presentation. On the question 'if you were asked to participate in a shared care program with the department of paediatric oncology for the follow-up of adult survivors of childhood cancer' 97\% (225/233) answered positive. About 64\% (150/233) of the GPs thought that participating would be a normal part of the care that GPs ought to provide. About $66 \%$ (154/233) of the GPs thought participating would not be very time consuming because they have only few adult paediatric cancer survivors in their practice. Seeing these patients as special (161/233) and to gain more knowledge were given as a reason to participate in approximately $70 \%$ $(164 / 233)$ of the GPs. Only seven GPs (3\%) refused.

Table 2 Content of questionnaire

\begin{tabular}{|c|c|}
\hline Questionnaire & Response rate $65 \%$ \\
\hline $\begin{array}{l}\text { Willing to participate in a shared care program with the department of paediatric } \\
\text { oncology for the follow-up of adult survivors of childhood cancer }\end{array}$ & $\begin{array}{l}\text { Yes } \\
\text { No }\end{array}$ \\
\hline \multicolumn{2}{|l|}{ Willing to participate in a shared care model for follow-up } \\
\hline Yes, because: & $\begin{array}{l}\text { Responsibility to be in charge for childhood cancer survivors } \\
\text { Few patients, less time consuming } \\
\text { Consider these patients as special } \\
\text { Gain more knowledge }\end{array}$ \\
\hline \multicolumn{2}{|l|}{ No, because (not specified) } \\
\hline $\begin{array}{l}\text { Compensation for GPs should depend on returning the records to improve the } \\
\text { motivation of GPs to return records }\end{array}$ & 7-point scale of importance \\
\hline Keep records used for screening simple & 7-point scale of importance \\
\hline Possibility of returning the forms electronically & 7-point scale of importance \\
\hline The practice assistant should be involved in the follow-up care & 7-point scale of importance \\
\hline $\begin{array}{l}\text { If you were asked to participate in a shared-care program for the follow-up } \\
\text { of adult childhood cancer survivors what would your requirements be? }\end{array}$ & Open-ended question \\
\hline $\begin{array}{l}\text { If you were asked to participate in a shared-care program for the follow-up } \\
\text { of adult childhood cancer survivors what would your reservations be? }\end{array}$ & Open-ended question \\
\hline
\end{tabular}


Figure 1 If you participated in follow-up what would be your requirements $(\%)$ ?

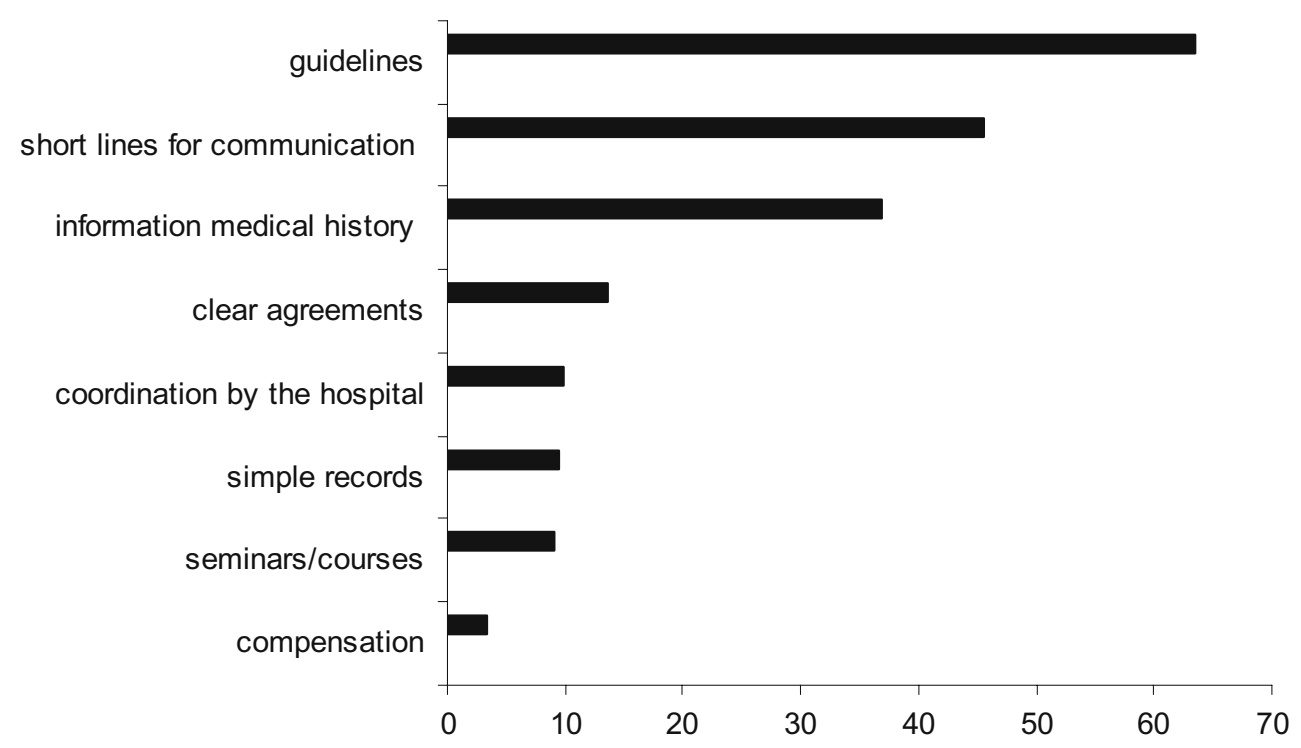

Reasons for not wanting to participate were; lack of time $(n=4)$, negative experience with collaboration with specialists in the past $(n=1)$, this is a task for the specialist $(n=1)$, and one GP did not give a reason for refusing to participate.

Approximately $37 \%(85 / 233)$ of the GPs thought that rewarding the GPs before they had returned the results of the screening to the department of oncology to improve the return rate was important, 40\% (93/233) thought this was not important, and 23\% (55/233) had no opinion. To keep the records simple was important for $96 \%(223 / 233)$ of the GPs. Almost half $(110 / 233,47 \%)$ of the GPs found it important that the forms could be returned electronically. Of the 206 GPs who had a practice assistant only $16 \%$ $(33 / 206)$ thought it was important that the practice assistant should be involved in the follow-up care and only $13 \%$ (27/206) thought it was important that the practice assistant should be responsible for returning the records of the screening to the LTFU clinic.

The two open questions were 'If you were asked to participate in a shared-care program for the follow-up of adult childhood cancer survivors what would your requirements be?' and 'If you were asked to participate in a shared-care program for the follow-up of adult childhood cancer survivors what would your reservations be?' The answers were categorized in seven respectively six different categories (Figs. 1 and 2).

Clear guidelines $(148 / 233,64 \%)$, short lines for communication $(106 / 233,45 \%)$, and clear information about
Figure 2 If you were asked to participate in follow-up what would be your objections (\%)?

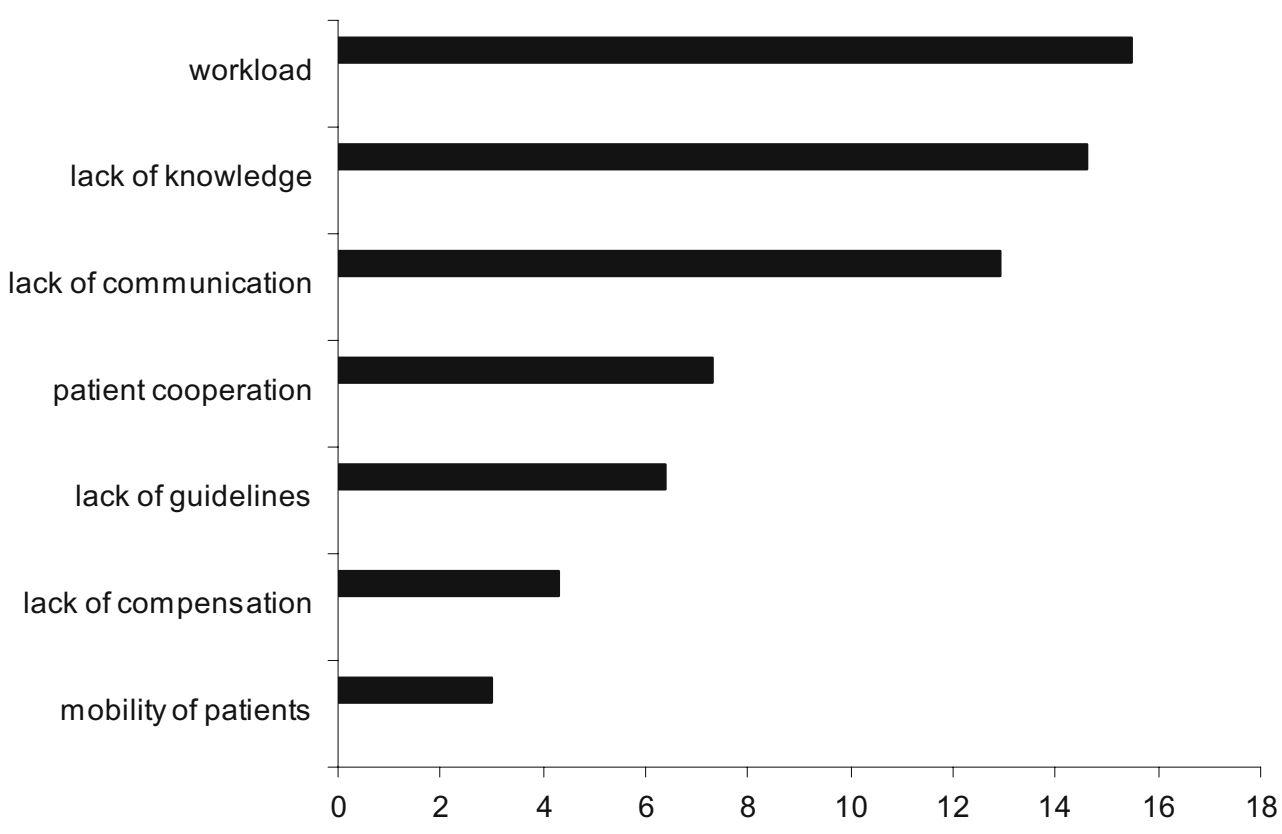


patient's medical history $(86 / 233,37 \%)$ are the main requirements for participation.

The main barriers to participate were: workload (36/233, $16 \%$ ), lack of knowledge $(34 / 233,15 \%)$ and lack of communication $(30 / 233,13 \%)$ between GP's and paediatric oncologists. Compensation was only a barrier to participate for $4 \%(10 / 233)$.

\section{Discussion}

There is consensus that the primary treatment of childhood cancer requires specialized care, and a plan for follow-up should be available for all survivors of childhood cancer. However, it is unclear whether oncology-based follow-up care is the appropriate model of care to meet long-term needs of survivors of childhood cancer. This study shows that adequate guidelines and medical information, as well as clear communication lines are important for GPs to participate in the long-term follow-up of adult childhood cancer survivors. These findings are confirmed in other studies $[6,7]$.

The combined efforts of paediatric oncologists and general practitioners will be important during the next decades to observe and report the life-long effects of treatment and lifestyle on survivors [8]. Models of shared care have been developed for chronic diseases such as diabetes, hypertension and asthma, and there are some examples of shared oncological care [9-11]. Some studies suggest that GPs are willing to become engaged in the follow-up care of cancer patients and that hospital followup provides no advantages compared to follow-up in primary settings $[12,13]$.

Because of the relative rarity of childhood cancer GPs lack knowledge of cancer related health risks [14]. This evaluation indicated that GPs appreciate postgraduate courses about adverse late effects, even though childhood cancer survivors represent a very small portion of individual GP practice. Whether actual practice behaviour changes following such a course should be studied in the future. Involving GPs in a shared-care model for the follow-up of adult childhood cancer survivors will increase the GP's knowledge for sequelae of cancer treatment in general and this could potentially benefit many types of cancer survivors. Shared care in oncology remains controversial. It is often promoted as offering patients care closer to home while, at the same time, reducing the burden on specialist services. GPs are divided on the issue, some viewing shared care as improving job satisfaction and others as another example of hospitals offloading work onto an already overloaded primary care sector. For the success of a shared care program it is important that GPs see the program as a step up from 'usual' care in general practice rather than a step down from hospital practice [15].

\section{Summary of main findings}

This study showed that the great majority of the attending GPs $(97 \%)$ are ready to participate in a shared care model for the long-term follow-up of adult paediatric cancer survivors and $64 \%$ of them state that participating would be a natural part of the care they provide.

It is interesting that when they were asked about their requirements in order to participate compensation was only important for $4 \%$ of the GPs. To make sure that GPs return the records of the screening to the LTFU clinic it is important to keep the records as simple as possible and it is worthwhile to consider if electronic exchange of information is possible as $47 \%$ of the GPs said that this way of exchange had their preference.

There seems not to be an important role for the practice assistant in the follow-up of adult survivors of paediatric cancer according to the views of GPs. Comments made about the role of the practice assistant were that they have a role in routine care for larger patient groups like diabetes but not for the special care these cancer survivors need.

\section{Limitations}

Because of the fact that most GPs are not acquainted with the issue of late effects in childhood cancer survivors, we decided to send the questionnaire to GPs who had been informed about this issue and about the shared care model as a possible model for the follow-up of adult childhood cancer survivors and not to representative groups from the entire country. The 1-week refresher course for GPs contains several health subjects and prior to registration attending GPs are not aware of the topics so they have no prior idea that adverse late effects in childhood cancer survivors would be one of the topics covered. From the two provinces where the study was conducted, $29 \%$ of GPs did not attend the course and therefore did not have the opportunity to participate. Furthermore $35 \%$ of those who attended the course did not complete the questionnaire and this might have led to a selection bias. Giving a course about late effects to the GPs prior to the completion of the questionnaire might have influenced the outcome positively. This could explain that the number of GPs willing to participate in the long-term follow-up of childhood cancer survivors $(97 \%)$ is much higher then the number of GPs willing to participate in the follow-up of patients with colorectal cancer $(50 \%)[6]$.

Although we cannot be sure whether our findings can be generalized for the entire Netherlands, there is no reason to believe that there will be significant differences in the attitudes of GPs in the North from those in the rest of the country. We also think that our findings go for countries 
where the health care system shows great resemblance to the Netherlands such as England, Flanders and the Scandinavian countries. We recognize the relative weakness of lack of validation of our questionnaire. By using open ended questions for the requirements and objections of participating we left the possibility for participants to individualize their own comments. We therefore believe that our questionnaire is reliable enough to represent the views of the GPs. Berendsen et al. [7] showed that 'developing personal relationships', 'gaining mutual respect' and 'increasing medical knowledge to the benefit of their patients' are the most important motivational factors for GPs for new collaboration models with medical specialists.

\section{Implications for future research or clinical practice}

We believe that follow-up care as a coordinated effort of the paediatric oncologist and the general practitioner could be used for the follow-up of adult childhood cancer survivors and should be further studied. Such a shared-care model could facilitate the necessary transition from paediatric-based care to adult care as childhood cancer survivors mature into adulthood. An example would be regular visits to the longterm follow-up clinic (LTFU) till the age of 18-21 years with transfer to a shared-care follow-up program in which the patients GPs are involved. The LTFU clinic has to support the GP continuously with specific guidelines and management of late effects and will see the patient on a low frequency base according to their individual risk profile. The corner stone of shared care is personal communication and provision of adequate guidelines.

We conclude that a significant number of GPs are ready to participate in the long-term follow-up of adult survivors of childhood cancer if guidelines and medical information provided and communication lines are clear; if they perform adequately needs further study.

Acknowledgement The corresponding author, Ria Blaauwbroek states that she had full access to all the data in the study and had final responsibility for the decision to submit for publication.

"All authors declare that they have nothing to declare"

\section{References}

1. Gatta G, Capocaccia R, Stiller C, Kaatsch P, Berrino F, Terenziani M. Childhood cancer survival trends in Europe: a EUROCARE Working Group study. J Clin Oncol. 2005;23 (16):3742-51.

2. Stevens MC, Mahler H, Parkes S. The health status of adult survivors of cancer in childhood. Eur J Cancer. 1998;34(5):694-8.

3. Blaauwbroek R, Stant AD, Groenier KH, Kamps WA, Meyboom B, Postma A. Health-related quality of life and adverse late effects in adult (very) long-term childhood cancer survivors. Eur J Cancer. 2007;43(1):122-30

4. Oeffinger KC, Mertens AC, Sklar CA, et al. Chronic health conditions in adult survivors of childhood cancer. N Engl J Med. 2006;355(15):1572-82.

5. Oeffinger KC. Childhood cancer survivors and primary care physicians. J Fam Pract. 2000;49(8):689-90.

6. Papagrigoriadis S, Koreli A. The needs of general practitioners in the follow-up of patients with colorectal cancer. Eur J Surg Oncol. 2001;27(6):541-4.

7. Berendsen AJ, Benneker WH, Schuling J, Rijkers-Koorn N, Slaets JP, Meyboom-de JB. Collaboration with general practitioners: preferences of medical specialists - a qualitative study. BMC Health Serv Res. 2006;6:155.

8. Aziz NM, Oeffinger KC, Brooks S, Turoff AJ. Comprehensive long-term follow-up programs for pediatric cancer survivors. Cancer. 2006;107(4):841-8.

9. Renders CM, Valk GD, de Sonnaville JJ, et al. Quality of care for patients with Type 2 diabetes mellitus - a long-term comparison of two quality improvement programmes in the Netherlands. Diabet Med. 2003;20(10):846-52.

10. Ciardullo AV, Daghio MM, Brunetti M, et al. Changes in longterm glycemic control and performance indicators in a cohort of type 2 diabetic patients cared for by general practitioners: findings from the "Modena Diabetes Project". Nutr Metab Cardiovasc Dis. 2003;13(6):372-6.

11. Nielsen JD, Palshof T, Mainz J, Jensen AB, Olesen F. Randomised controlled trial of a shared care programme for newly referred cancer patients: bridging the gap between general practice and hospital. Qual Saf Health Care. 2003;12(4):263-72.

12. Worster A, Bass MJ, Wood ML. Willingness to follow breast cancer. Survey of family physicians. Can Fam Physician. 1996;42:263-8.

13. Grunfeld E, Fitzpatrick R, Mant D, et al. Comparison of breast cancer patient satisfaction with follow-up in primary care versus specialist care: results from a randomized controlled trial. $\mathrm{Br} \mathrm{J}$ Gen Pract. 1999;49(446):705-10.

14. Landier W, Wallace WH, Hudson MM. Long-term follow-up of pediatric cancer survivors: education, surveillance, and screening. Pediatr Blood Cancer. 2006;46(2):149-58.

15. Maher EJ, Millar D. Shared care: step down or step up? Qual Saf Health Care. 2003;12(4):242. 